\title{
Expiratory Time
}

National Cancer Institute

\section{Source}

National Cancer Institute. Expiratory Time. NCI Thesaurus. Code C120932.

The recorded time interval between the initiation of exhalation and the time at which no further lung volume reduction occurs. 